\title{
The Evaluation of Timing Performance for Different Smartphone Devices via Single Server
}

\author{
Miran Baban $^{1}$ and Hozan Fadhil ${ }^{2}$
}

\begin{abstract}
These instructions give you guidelines for preparing papers for conferences or journals. Use this document as a template if you are using Microsoft Word. Otherwise, use this document as an instruction set. The electronic file of your paper will be formatted further at ICEHM. Define all symbols used in the abstract. Do not cite references in the abstract. Do not delete the blank line immediately above the abstract; it sets the footnote at the bottom of this column. Page margins are $1,78 \mathrm{~cm}$ top and down; $1,65 \mathrm{~cm} \mathrm{left}$ and right. Each column width is $8,89 \mathrm{~cm}$ and the separation between the columns is $0,51 \mathrm{~cm}$.
\end{abstract}

Keywords - Search Engine, Database, Networking System, Local Area Network, Wide Area Network.

\section{INTRODUCTION}

C ALCULATING devices response is a way to show its performance while connected to the network, LAN or WAN. LAN such as a network that is established in a small organization, private or public. This network is to connect the computing machines to exchange the information between employees of an organization. Thus, reduce the labor work that is needed to accomplish the work otherwise. While WAN is to connect servers to the web together, such as exchanging e-mails. This research works on the LAN using three mobile devices (phones, Windows phones, and smartphones). These devices connected to a computer server that includes a work page with search engine feature that is used to tell the access time for each smartphone.

Each device that connects to a network (LAN or WAN) needs a different amount of time to reach the destination, and this is due to different architecture and capacity storage. Also, any connection between devices, there will be an amount of lost packet in the connection.

The idea behind this study is to measure the amount of time elapsed by these three devices. Each device gives different results in milliseconds. Also, detecting the number of packets during the connection.

The expected outcome of this research is getting different amounts of time during network connection and identifying some lost packets in the same process.

\footnotetext{
${ }^{1}$ Affiliation (Iraq-KRG (Kurdistan Region) University of Sulaimani, miran.mohammed@univsul.edu.iq)

${ }^{2}$ Affiliation (Iraq-KRG (Kurdistan Region) Sulaimani Pol
}

Each device that connects to a network (LAN or WAN) needs a different amount of time to reach the destination, and this is due to different architecture and capacity storage. Also, any connection between devices, there will be an amount of lost packet in the connection.

The idea behind this study is to measure the amount of time elapsed by these three devices. Each device gives different results in milliseconds. Also, detecting the number of packets during the connection.

The expected outcome of this research is getting different amounts of time during network connection and identifying some lost packets in the same process.

The content design of this study is as follows:

- Introduction: In this section, a general definition will be introduced about the paperwork and shows the main idea of the result.

- Background research: Presents some academic papers from the international journal and shows some real aspects of the issue.

- Related work: Investigating some academic research that is relevant to this research.

- Design implementation: In this section, research discusses the steps of developing the system.

- Result and Discussion: In this part, it shows the final output of the research.

- Conclusion: It summarizes all ideas that are presented in this study.

- Future work: Illustrates some possibilities to expand this study to comprise more advanced ideas related to network communication.

\section{BACKGROUND AND RESEARCH}

\subsection{Network Connections}

Computers including desktop and laptop, are connected among each other via a connection mechanism called Network techniques. The Same thing can be found on any other devices that have capabilities of connection and exchange data (e.g. texts messages, sound, pictures, and videos). There are many ways for connection creation, for instance, connections via LAN (Local Area Network), WAN 
(Wide Area Network) [1] [2].

\subsubsection{LAN (Local Area Network)}

It is an example of network connections among computers and different electronic devices that are located in the small place, for instance, an organization or small private sectors or government sectors. In this type of connection, the computer is connected to an intranet manner and client can exchange basic data that are related to organizations that they work [3].

\subsubsection{WAN (Wide Area Network)}

In this network connection, the computer is connected to the larger network that comprises all equipment all over the world which is connected to servers remotely and from other countries. Wider users participate in this connection type and for different purposes, for example, internet browsing, email checking, social media, research and education and business and shopping. Also, WAN can provide easy access to any knowledge that user want to access and easy communication with others [4].

\subsection{Mobile Device}

In this network connection, the computer is connected to the larger network that comprises all computer all over the world which is connected to servers remotely and from other countries. Wider users participate in this connection type and for different purposes, for example, internet browsing, email checking, social media, research and education and business and shopping. Also, WAN can provide easy access to any knowledge that user want to access and easy communication with others [5].

\subsection{Smart Phone}

The newest generation that the technologies saw over the past few years ago, which is the development of mobile production from few features components to the most modern properties that help users to get their daily needs with lots of facilities easily. Also, there are numbers of smartphones types, each of them has its company that they product, for example, Apple, Samsung. And these smartphones have their modern operating systems (Apple and Android) [6].

\subsection{Hardware Performance}

This term refers to understand the capabilities and performance that each hardware device can perform during their connections with network either LAN or WAN. Also, this performance can be tested by checking the speed of response time. Thus, it can result in that which device can perform faster and more efficient [7].

\subsection{Computer Server}

It is obvious that in each networking system either locally or widely there are some servers' works on managing requested data from clients and response they base upon their required data. In fact, servers provide the necessary security of a system that customers work on, for instance in some organizations some systems provide some level of safety for using the database [8].

\subsection{Media Access Control (MAC)}

Is a sublayer and it is important in LANs networking, particularly wireless ones because wireless is naturally a broadcast channel. MAC is a physical address that assign for each device such as computers and mobile devices, and it is located in the data link layer of the network hierarchy.

This address is used as a main part of the communication system, especially in the LAN networks. The reason for using MAC address in the network is to recognize each device as a unique entity on the network [9].

\subsection{Network Layer}

The network layer is concerned with getting packets from the source all the way to the destination. Getting to the destination may require making many hosts at intermediate routers along the way. This function clearly contrasts with that of the data link layer, which has the most modest goal of just moving frames from one end of the wire to the other. Thus, the network layer is the lowest layer that deals with the end-to-end transmission [10].

\subsection{Search Engine}

Search engine nowadays there are lots of technologies have been proposed for the web as new properties that internet users to use for daily purposes. One of these features is called Search Engine. This technique becomes one of the essential accessories that help man to find a piece of information that they look for on the Web. Although there are lots of information on the full net on different websites, when users search for a particular one, will get a result in an organized way. Thus, search technology becomes as an initial prototype that each website must include it.

Also, there are different methods of using a search engine. These techniques help find the necessary information in the more accurate way if it is employed. For instance, a user requires finding a keyword (physical layer) and (network layer) [11].

In this case, it is necessary to use the "+" sign between the two keywords. In contrast, if he needs to get one of the keywords and not the other one it is recommended to use the “_. sign [12].

\section{RELATED WORK}

During the study, some new researches have been conducted with some identity idea with this reach, this due to emphasizes the research thoughts and give more examples.

According to (Srirama, et.al) found some new ideas about mobile web performance, and during their research and study, they have found new results regarding their data and foundation.

In fact, they tested the performance of mobile devices including smartphone and all other telephones that they have abilities to connect with Internet services via wireless. Also, they have used and tested some methodologies, and one of these methodologies that they discussed on in a more long way is the WS-wireless technologies. And this for providing 
security reason while connecting to the mobile and sending data between other devices, for example connecting to the social website and chatting websites.

Their study also comprises the security environment for sending messages between mobile devices. Also, they mentioned some other idea that can have an impact on the safety purposes on different mobile hardware and architectures while they are in communication with other devices. The following are the test result their researchers have found them.

TABLE I

Message VARIATION (IN Byte) With SECURITy (SRIRAMa, ET.AL)

\begin{tabular}{|c|c|c|c|c|}
\hline $\begin{array}{c}\text { Original } \\
\text { message size }\end{array}$ & 1024 & 2048 & 5120 & 10240 \\
\hline $\begin{array}{c}\text { Message size } \\
\text { with Signature }\end{array}$ & 1726 & 2750 & 5822 & 10942 \\
\hline $\begin{array}{c}\text { Encrypted } \\
\text { message size }\end{array}$ & 1804 & 3168 & 7264 & 14092 \\
\hline $\begin{array}{c}\text { Secured } \\
\text { message size }\end{array}$ & 2611 & 3975 & 8071 & 14899 \\
\hline
\end{tabular}

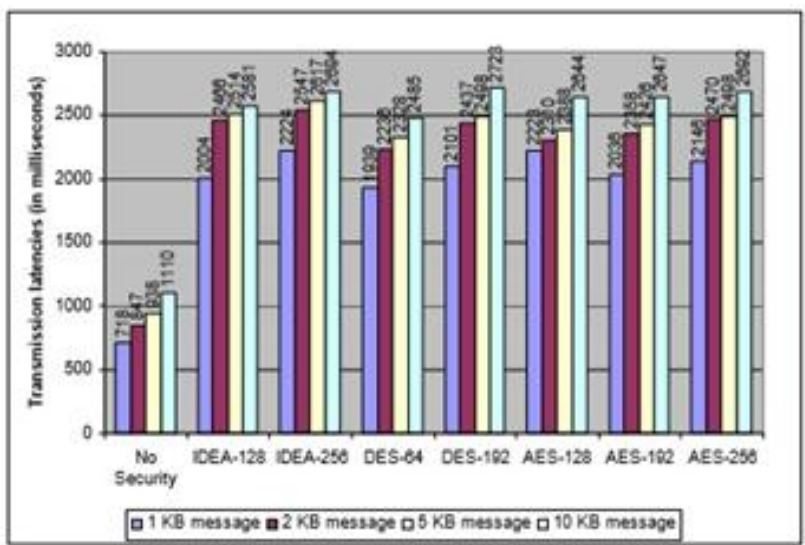

Fig. 1: Symmetric algorithm encryption with RSA

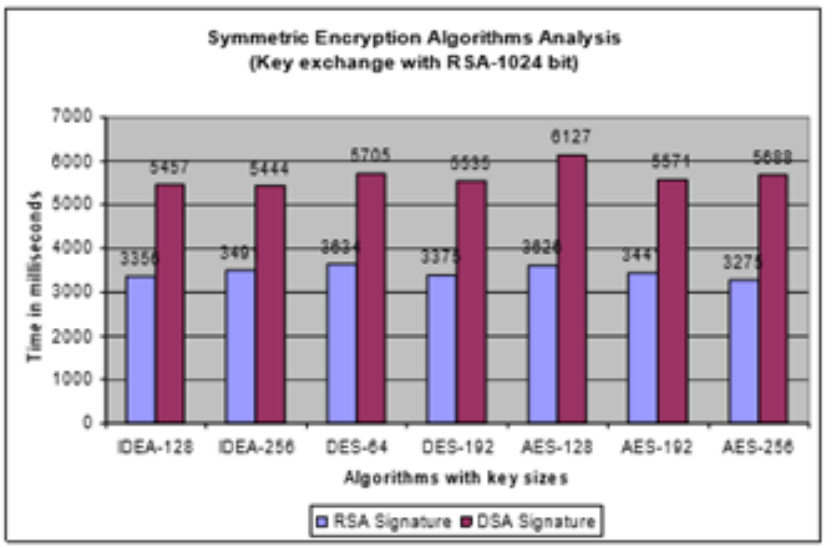

Fig. 2: Encryption Performance with latencies.

However, another study that related to this research is the work of (Gassone, Elia, and et.al); they discuss the performance of web access speed time and the response of the server from computer devices. They consider the traffic and the way of connected computers and server may have a noticeable impact on the access speed. Also, they show the better way for network communication which can provide more speed for their clients while requesting a page from the server.

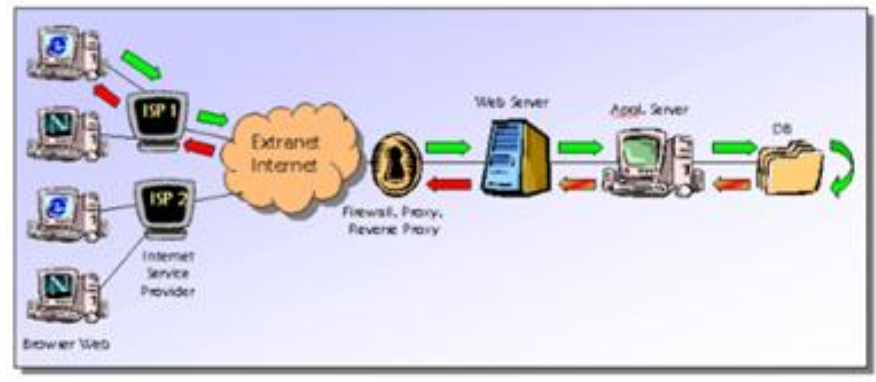

Fig.3: System architect for client server.

Also, they introduced a new method that can measure the amount of time and capacity that will take each client to access the server for getting data or any knowledge that will be required by the user. And they use BMPOP technique to evaluate the data.

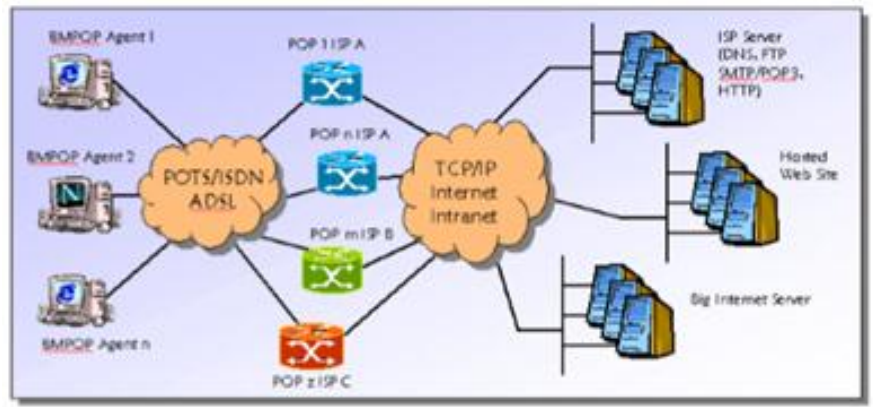

Fig.4: BMPOP measuring transaction

Another study which held by (Miran Baban, et.al) on 2013, which is about the same idea for this study, but the difference is that the researchers in their study depended on evaluating the computer speed performance according to their different architecture with different windows and apache server. While this study is about using the different mobile version with different mobile hardware architectures 64 and 32 bits. Finally, they have come into account and got the following data as the research final result.

TABLE II

64-BIT ARCHITECTURE PERFORMANCE EVALUATION

\begin{tabular}{|c|l|l|l|}
\hline Hardware & Platform & $\begin{array}{l}\text { Response- } \\
\text { time }\end{array}$ & IIS version \\
\hline 64 bits & Windows Server 2003 & $1.645 \mathrm{Sec}$ & 7 \\
\hline 64 bits & Windows XP & $1.004 \mathrm{Sec}$ & 5.1 \\
\hline 64 bits & Windows 7 & $1.039 \mathrm{Sec}$ & 7 \\
\hline
\end{tabular}

32-BIT ARCHITECTURE PERFORMANCE EVALUATION

\begin{tabular}{|l|l|l|l|}
\hline Hardware & Platform & $\begin{array}{l}\text { Response- } \\
\text { time }\end{array}$ & IIS version \\
\hline 32 bits & Windows Server 2003 & $1.046 \mathrm{Sec}$ & 7 \\
\hline 32 bits & Windows XP & $0.005 \mathrm{Sec}$ & 5.1 \\
\hline 32 bits & Windows 7 & $1.032 \mathrm{Sec}$ & 7 \\
\hline
\end{tabular}




\section{IMPLEMENTATION AND DESIGN}

This research has been done by using software and hardware as requirement components such as Apache server that provide a server function and response users request, especially when user ask for a page. And this server will calculate the amount of time to execute and response time from the user mobile. Also, in this research, a three mobiles have been used as a way to simulate that users use mobile to access a web page on a server. And the last thing that has been used is a server, which a computer laptop.

\section{V.RESULT AND DISCUSSION}

The result and outcome of this research after conducting some tests on the server while connecting three different mobile architectures and different smart phone versions. While the three mobiles are accessing the server for requesting a web page, and each mobile shows different amount of time for speed access with different run time execution. For each mobile we have showed the hardware architecture and their speed time for requesting a specific web page on the particular server.

TABLE IV

NOKIA LUMIa 920 SMARTPHONE TESTING PERformanCE

\begin{tabular}{|l|l|}
\hline Name & Windows Phone \\
\hline Model & Nokia Lumia 920 \\
\hline MAC address & A8-44-81-C1-0F-8C \\
\hline IMEI & 354149056574392 \\
\hline $\begin{array}{l}\text { Time required to access server } \\
\text { (in milliseconds) }\end{array}$ & 0.1349449157 \\
\hline
\end{tabular}

TABLE V

SAMSUNG GALAXY S6 SMARTPHONE TESTING PERFORMANCE

\begin{tabular}{|l|l|}
\hline NAME & SAMSUNG GALAXY S6 \\
\hline MODEL NO. & SM-G920F \\
\hline ANDROID VERSION & 5.1 .1 \\
\hline BASEBAND VERSION & G920FXXV3COI9 \\
\hline KERNEL VERSION & $3.10 .61-5917605$ \\
\hline $\begin{array}{l}\text { TIME REQUIRED TO ACCESS } \\
\text { SERVER (IN MILLISECONDS) }\end{array}$ & 1.05142 \\
\hline
\end{tabular}

TABLE VI

IPHONE-5 SMARTPHONE Testing PeRformance

\begin{tabular}{|l|l|}
\hline Name & iPhone 5 \\
\hline Model & iPhone 5 \\
\hline $\begin{array}{l}\text { Time required to access } \\
\text { server (in milliseconds) }\end{array}$ & 900.25 \\
\hline
\end{tabular}

\section{CONCLUSION}

In this paper, we studied the timing performance of different mobile stations while accessing a single server. We observed that there were a considerable amount of difference in the access time between the various devices. It is due to the different hardware architecture employed in each one in addition to the difference in the operating systems employed in each one. We observe that the Samsung Galaxy smartphone was the fastest one to access the server, proceeded by the Nokia and then the iPhone. And this is due to the hardware features of the Samsung Galaxy given in Tables (IV, V, and VI).

\section{FUTURE WORK}

In this study, we concentrated on the evaluation of the performance of different mobile devices technology (different handsets and operating systems) for accessing a web page search engine to calculate the access time of the resulting searches. However, we have plans to widen our work to comprise the calculation of lost packets during accessing the server.

\section{ACKNOWLEDGMENT}

We would like to thank everyone who helped us to conduct this study via different facilities including places and opportunities. Also, I would like to give a special thanks to my well-known academic friend, Hozan Nihad Mohammed Fadhil.

\section{REFERENCES}

[1] R. Bassily and S. Ulukus, "Secure communication in multiple relay networks through decode-and-forward strategies", Journal of Communications and Networks, vol. 14, no. 4, pp. 352-363, 2012.

[2] J. Rapinski and S. Cellmer, "Analysis of Range Based Indoor Positioning Techniques for Personal Communication Networks", Mobile Networks and Applications, 2015.

[3] H. Lee, K. Park, Y. Ko and C. Choi, "Wireless LAN with medical-grade QoS for e-healthcare", Journal of Communications and Networks, vol. 13, no. 2, pp. 149-159, 2011.

[4] F. Dressler, A. Misra and R. Shorey, "Special Issue: Recent Advances in Wireless Communication Systems", Mobile Networks and Applications, vol. 16, no. 5, pp. 586-588, 2011.

[5] R. Muhlberger, "Mobile devices as distributed database components for relocating applications", International Journal of Mobile Communications, vol. 2, no. 3, p. 285, 2004.

[6] Y. Jang, S. Chang and Y. Tsai, "Smartphone security: understanding smartphone users' trust in information security management", Security and Communication Networks, vol. 7, no. 9, pp. 1313-1321, 2013.

[7] V. Ravi, R. Shankar and M. Tiwari, "Productivity improvement of a computer hardware supply chain", Int J Productivity \& Perf Mgmt, vol. 54, no. 4, pp. 239-255, 2005.

[8] R. Rose and I. Arizmendi, "Efficient client-server based implementations of mobile speech recognition services", Speech Communication, vol. 48, no. 11, pp. 1573-1589, 2006.

[9] M. Baban, "UNIVERSITY SEMINARS ATTENDANCE CHECKING SYSTEM USING QR CODE IMAGE SCANNER", International Journal of Advance Research, vol. 3, no. 8, 2015.

[10] J. Wang, J. Lai, Y. Liu and W. Deng, "Network-layer abstraction and simulation of vehicle communication stack", Wireless Netw, vol. 21, no. 3, pp. 709-725, 2014.

[11] C. Argenton and J. Prufer, "Search Engine Competition with Network Externalities", SSRN Electronic Journal.

[12] K. Eliaz and R. Spiegler, "A Simple Model of Search Engine Pricing*", The Economic Journal, vol. 121, no. 556, pp. F329-F339, 2011. 